\title{
LMI based antiswing adaptive controller for uncertain overhead cranes
}

\author{
Nga Thi-Thuy Vu \\ Hanoi University of Science and Technology, Vietnam
}

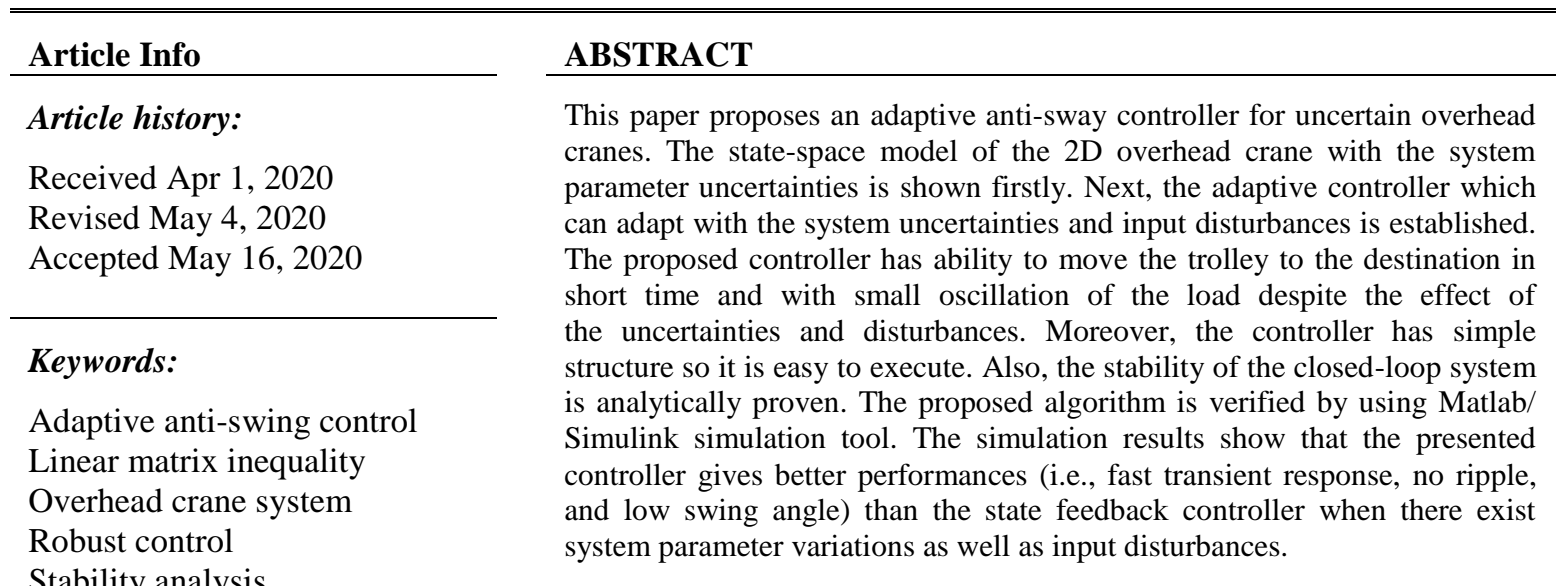

Copyright $@ 2020$ Institute of Advanced Engineering and Science. All rights reserved.

\section{Corresponding Author:}

Nga Thi-Thuy Vu,

School of Electrical Engineering,

Hanoi University of Science and Technology, Vietnam.

Email: nga.vuthithuy@hust.edu.vn

\section{INTRODUCTION}

The overhead cranes which are widely used for transporting heavy loads are one of the most popular underactuated mechanical systems in that the number of the actuators is less than the degree of freedom. The deficiency of actuator for sway dynamics presents a coupling effect between the load sway motion and the trolley traveling motion. The transient swing of payload may cause a safety hazard to employees, transferred goods and surrounding objects. In addition, the lack of actuator makes the control design of the underactuated system much more difficult than the full actuated systems. For this reason, designing the controller for the overhead crane system which can move the trolley to the destination as fast as possible with acceptable swing angle attracts the consideration of many researchers.

Nowadays, there have been various control methods that can guarantee the good performance for the overhead crane systems both in open loop and closed loop. In the class of open loop control, the swing of payload is abolished by some approaches such as input shaping [1-4], trajectory planning [5, 6]. However, in general, the open-loop control system can not guarantee the good performance in the case of system uncertainties and external disturbances. Therefore, many closed-loop control techniques are applied to the overhead crane system to improve the performance such as nonlinear feedback [7-11], partial feedback linearization [12, 13], fuzzy logic control [14-17], sliding mode control [18-21] and so on.

It is widely recognized that adaptive control method has the advantage of handing with uncertain systems. In the field of overhead crane control, the adaptive control technique is also considered by some researchers. In [22] the fuzzy logic controller is used to keep the system stable and an adaptive algorithm is provided to tune the free parameters. The given strategy is simple but robust to the variation of the system parameters (wire length and payload weight) as well as external disturbances. However, the stability of overall system is not presented. In [23], a fuzzy sliding-mode control is designed for the antisway trajectory 
tracking of the nominal plant. Then, a fuzzy uncertainty observer is used to cope with system uncertainties as well as actuator nonlinearities. This observer is incorporated with the fuzzy sliding-mode control law for the development of the adaptive fuzzy sliding-mode controller. This scheme guarantees asymptotic stability and robust performance but it is quite complicated. An adaptive sliding-mode antisway control of uncertain overhead cranes with high-speed hosting motion is shown in [24]. In this scheme, the asymptotic stability of the sway dynamic is achieved by the sliding-mode controller, the system uncertainties is coped by a fuzzy observer. This algorithm gives the robust antisway performance to overhead cranes regardless of hosting velocity and system uncertainties. The stability of the system, however, is proven in analysis and simulation only.

This paper proposed an antisway adaptive controller for overhead cranes. In particular, the model of the overhead crane is built in the form of state-space at first. Then, the adaptive controller with feedforward and feedback components is introduced. This controller has the ability to drive the trolley to the target with high speed and low swing angle. Also, the proposed controller can remove the effect of the parametric uncertainties as well as the input disturbances. Moreover, the structure of the controller is not complicate and this leads to simplify in the execution. The stability of the overall system is guaranteed by the Lyapunov theory. Finally, the simulation is executed by Matlab/Simulink for both proposed adaptive controller and conventional state feedback controller. The simulation results indicate that the suggested controller gives the good performance, i.e., fast response, no steady state error, no payload swing angle even under the condition of system uncertainties. The main contribution of this research work can be cited as the following:

- The proposed controller can drive the trolley to the target with fast responses and almost no swing angle.

- The scheme works well under the effect of rope length, variation load mass, the external disturbances.

- In comparision with the existing works which solve the same problems of the overhead crane systems, the presented controller has simple structure and the stability is proven via Lyapunov theory by using the Linear Matrix Inequality.

\section{SYSTEM MODEL AND LMI BASED ADAPTIVE CONTROLLER DESIGN}

Figure 1 describes the block diagram of an overhead crane. The trolley moves along the horizontal axis (Ox-axis) with its load which is hung at the end of the rope.

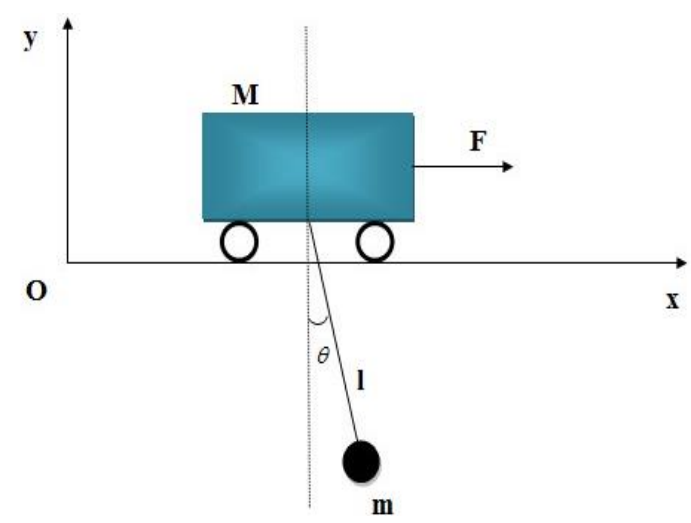

Figure 1. Block diagram of an overhead crane system

The motion equation of the overhead crane is given as the following [25]:

$$
\left\{\begin{array}{c}
(M+m) \ddot{x}+m l \ddot{\theta} \cos \theta-m l \dot{\theta}^{2} \sin \theta=u \\
l \ddot{\theta}+g \sin \theta+\ddot{x} \cos \theta=0
\end{array}\right.
$$

where

$M$ : trolley mass $[\mathrm{kg}]$

$l$ : cable length $[\mathrm{m}]$

$g$ : gravity acceleration $\left[\mathrm{m} / \mathrm{s}^{2}\right]$

$m$ : payload mass $[\mathrm{kg}]$

$x$ : trolley position $[\mathrm{m}]$

$\theta$ : payload swing angle [deg]

$u$ : control input corresponding to control force exerted on the trolley [N] 
The model (1) can be rewritten as:

$$
\left\{\begin{array}{l}
\ddot{x}=\frac{m g \sin \theta \cos \theta+m l \dot{\theta}^{2} \sin \theta}{M+m \sin ^{2} \theta}+\frac{1}{M+m \sin ^{2} \theta} u \\
\ddot{\theta}=-\frac{(M+m) g \sin \theta+m l \dot{\theta}^{2} \sin \theta \cos \theta}{\left(M+m \sin ^{2} \theta\right) l}-\frac{\cos \theta}{\left(M+m \sin ^{2} \theta\right) l} u
\end{array}\right.
$$

It should be noted that since the sway angle is small, i.e. it is desired to be zero, then $\cos \theta \approx 1, \sin \theta \approx \theta$, and $\theta^{2} \approx 0$. The model (2) can be simplified as:

$$
\left\{\begin{array}{l}
\ddot{x}=\frac{m g \theta+m l \dot{\theta}^{2} \theta}{M}+\frac{1}{M} u \\
\ddot{\theta}=-\frac{(M+m) g \theta+m l \dot{\theta}^{2} \theta}{M l}-\frac{1}{M l} u
\end{array}\right.
$$

Defining the state variables:

$$
X=\left[\begin{array}{llll}
x_{1} & x_{2} & x_{3} & x_{4}
\end{array}\right]^{T}=\left[\begin{array}{llll}
x-x_{d} & \dot{x}-\dot{x}_{d} & \theta-\theta_{d} & \dot{\theta}-\dot{\theta}_{d}
\end{array}\right]^{T}
$$

where $x_{d}$ and $\theta_{d}$ are the desired values of $x$ and $\theta$, respectively.

With this definition and by using the fact that the $x_{d}$ and $\theta_{d}$ do not change suddenly in a short sampling interval, the system model (3) can be rewritten as:

$$
\left\{\begin{array}{l}
\dot{x}_{1}=x_{2} \\
\dot{x}_{2}=k_{1} x_{3}+k_{2} x_{3} x_{4}^{2}+k_{3} u \\
\dot{x}_{3}=x_{4} \\
\dot{x}_{4}=k_{4} x_{3}+k_{5} x_{3} x_{4}^{2}+k_{6} u
\end{array}\right.
$$

where $k_{1}=\frac{m g}{M}, k_{2}=\frac{m l}{M}, k_{3}=\frac{1}{M}, k_{4}=-\frac{(M+m) g}{M l}, k_{5}=-\frac{m}{M}, k_{6}=-\frac{1}{M l}$

The control input $u$ can be separated into two parts, $u_{1}$ and $u_{2}$, where $u_{1}$ is the feedback control component which stabilizes the error dynamics of the system and $u_{2}$ is the nonlinearity compensating control component given as

$$
u_{2}=-x_{3} x_{4}^{2}
$$

In considering the system parameter uncertainties, the model (4) becomes:

$$
\left\{\begin{array}{l}
\dot{x}_{1}=x_{2} \\
\dot{x}_{2}=\left(k_{1}+\Delta k_{1}\right) x_{3}+\left(k_{2}+\Delta k_{2}\right) x_{3} x_{4}^{2}+\left(k_{3}+\Delta k_{3}\right)\left(u_{1}+u_{2}\right) \\
\dot{x}_{3}=x_{4} \\
\dot{x}_{4}=\left(k_{4}+\Delta k_{4}\right) x_{3}+\left(k_{5}+\Delta k_{5}\right) x_{3} x_{4}^{2}+\left(k_{6}+\Delta k_{6}\right)\left(u_{1}+u_{2}\right)
\end{array}\right.
$$

where $\Delta k_{i}(i=1$ to 6$)$ are the uncertainties of $k_{i}$. It does not lose the generation with the assumption that $\Delta k_{3}$ and $\Delta k_{6}$ are not only the uncertainties of $k_{3}$ and $k_{6}$ but also include the input disturbances and error in the feedforward channel represented by $\delta$. With this assumption, the model (6) becomes:

$$
\left\{\begin{array}{l}
\dot{x}_{1}=x_{2} \\
\dot{x}_{2}=\left(k_{1}+\Delta k_{1}\right) x_{3}+k_{3} u_{1}+k_{3} \delta\left(u_{1}+x_{3} x_{4}^{2}\right) \\
\dot{x}_{3}=x_{4} \\
\dot{x}_{4}=\left(k_{4}+\Delta k_{4}\right) x_{3}+k_{6} u_{1}+k_{6} \delta\left(u_{1}+x_{3} x_{4}^{2}\right)
\end{array}\right.
$$

The model (7) can be rewritten in the state-space form as

$$
\dot{x}=(A+\Delta A) x+B\left[u_{1}+\delta f(x, u)\right]
$$


where

$$
\begin{aligned}
& A=\left[\begin{array}{cccc}
0 & 1 & 0 & 0 \\
0 & 0 & k_{1} & 0 \\
0 & 0 & 0 & 1 \\
0 & 0 & k_{4} & 0
\end{array}\right], \Delta A=\left[\begin{array}{cccc}
0 & 0 & 0 & 0 \\
0 & 0 & \Delta k_{1} & 0 \\
0 & 0 & 0 & 0 \\
0 & 0 & \Delta k_{4} & 0
\end{array}\right] \\
& B=\left[\begin{array}{llll}
0 & k_{3} & 0 & k_{5}
\end{array}\right]^{T}, f(x, u)=u_{1}+x_{3} x_{4}^{2}
\end{aligned}
$$

In which $\Delta A$ component expresses the uncertainties of the system parameters parameters. Assume that there exists a positive definite matrix $P \in R^{4 \times 4}$ satisfying the following inequality

$$
(A+\Delta A)^{T} P+P(A+\Delta A)+Q-2 P B R^{-1} B^{T} P<0
$$

where $Q \in R^{4 \times 4}$, and $R \in R^{2 \times 2}$ are positive definite matrices. Assume the controller $K$ is given by:

$$
K=R^{-1} B^{T} P
$$

and the adaptive law

$$
\dot{\delta}_{e s}=\gamma f(x, u) x^{T} P B, \quad \gamma>0
$$

where $\delta_{\text {es }}$ is the estimated value of $\delta$.

Consider the following theorem:

Theorem: Assume that the LMI condition (9) is feasible for some $P$ and the controller gain $K$ is given by (10), the adaptive law is given by (11). Then the controller $u_{1}$ can make the error dynamics $x$ converge to zero.

$$
u_{1}=-K x-\delta_{e s} f(x, u)
$$

Proof: Let us choose the Lyapunov function as

$$
V=x^{T} P x+\delta_{e}^{2} \gamma^{-1}
$$

where $\delta_{e}=\delta_{e s}-\delta$. Its time derivative along the error dynamics (11) is given by

$$
\begin{aligned}
\dot{V} & =2 x^{T} P \dot{x}+2 \delta_{e} \dot{\delta}_{e} \gamma^{-1} \\
& =2 x^{T} P[(A+\Delta A) x+B u+B \delta f(x, u)]+2 \delta_{e} \gamma^{-1}\left(\dot{\delta}_{e s}-\dot{\delta}\right) \\
& =2 x^{T} P\left[(A+\Delta A) x+B\left(-K x-\delta_{e s} f(x, u)\right)+B \delta f(x, u)\right]+2 \delta_{e} \gamma^{-1} \dot{\delta}_{e s} \\
& =2 x^{T} P[(A+\Delta A)-B K] x-2 x^{T} P B \delta_{e s} f(x, u)+2 x^{T} P B \delta f(x, u)+2 \delta_{e s} \gamma^{-1} \dot{\delta}_{e s} \\
& =2 x^{T} P[(A+\Delta A)-B K] x-2 x^{T} P B \delta_{e s} f(x, u)+2 \delta_{e s} f(x, u) x^{T} P B \\
& =2 x^{T} P[(A+\Delta A)-B K] x
\end{aligned}
$$

The LMI condition (9) implies that

$$
\dot{V}<-x^{T} Q x \leq 0
$$

Then, by integrating both sides of (15), the following equation is derived

$$
\int_{0}^{\infty} x(\tau)^{T} Q x(\tau) d \tau=-\int_{0}^{\infty} \dot{V}(\tau) d \tau=V(0)-V(\infty)<\infty
$$

This implies $x \in L_{2} \cap L_{\infty}, \delta \in L_{\infty}$. Combining the previous results and using Barbalat's lemma, $x$ converges to zero as time goes to infinity, that is,

$$
\lim _{t \rightarrow \infty} x(t)=0
$$


Remark 1: The equation of $\Delta A$ can be rewritten as the following form:

$$
\Delta A=\left[\begin{array}{cccc}
0 & 0 & 0 & 0 \\
0 & 0 & \Delta k_{1} & 0 \\
0 & 0 & 0 & 0 \\
0 & 0 & \Delta k_{4} & 0
\end{array}\right]=E F \Delta k_{1}+G F \Delta k_{4}
$$

where

$$
E=\left[\begin{array}{ll}
0 & 0 \\
1 & 0 \\
0 & 0 \\
0 & 0
\end{array}\right], F=\left[\begin{array}{ll}
0 & 0 \\
0 & 0 \\
1 & 0 \\
0 & 0
\end{array}\right]^{T}, G=\left[\begin{array}{ll}
0 & 0 \\
0 & 0 \\
0 & 0 \\
1 & 0
\end{array}\right]
$$

The inequality (9) is rewritten as

$$
A^{T} P+P A-2 P B R^{-1} B^{T} P+Q+\triangle A^{T} P+P \triangle A<0
$$

The above inequality (19) is satisfied if the following inequality holds for some positive $\rho$

$$
A^{T} P+P A+Q-2 P B R^{-1} B^{T} P+\rho P E E^{T} P+\frac{1}{\rho} F^{T} F \Delta k_{1}^{2}+\rho P G G^{T} P+\frac{1}{\rho} F^{T} F \Delta k_{4}^{2}<0
$$

where the following inequality is used

$$
\begin{aligned}
\Delta A^{T} P+P \Delta A & =\Delta k_{1} F^{T} E^{T} P+\Delta k_{1} P E F+\Delta k_{4} F^{T} G^{T} P+\Delta k_{4} P G F \\
& \leq \rho P E E^{T} P+\frac{1}{\rho} F^{T} F \Delta k_{1}^{2}+\rho P G G^{T} P+\frac{1}{\rho} F^{T} F \Delta k_{4}^{2}
\end{aligned}
$$

Assume that $\left|\Delta k_{1}\right| \leq \zeta$ and $\left|\Delta k_{4}\right| \leq \zeta$ for some known positive constant $\zeta$, then inequality (20) is satisfied if the following Riccati-like inequality has a positive definite solution matrix $P \in R^{4 \times 4}$ :

$$
A^{T} P+P A+Q-2 P B R^{-1} B^{T} P+\rho P E E^{T} P+\rho P G G^{T} P+\frac{2}{\rho} \zeta^{2} F^{T} F<0
$$

Remark 2: By using the Schur complement formula, it can be shown that the Riccati-like inequality (21) is equivalent to the following linear matrix inequality (LMI)

$$
X>0, \quad\left[\begin{array}{ccc}
A X+X A^{T}-2 B R^{-1} B^{T}+\rho E E^{T}+\rho G G^{T} & X & \zeta X F^{T} \\
X & -Q^{-1} & 0 \\
\zeta F X & 0 & -\frac{\rho}{2} I
\end{array}\right]<0
$$

Thus, by solving the above simple LMI and setting $P=X^{-1}$, we can easily obtain the positive definite solution matrix $P$ of (21).

\section{CONTROL STRATEGY VERIFICATION}

In order to validate the effectiveness of the proposed adaptive antiswing controller, the simulation and experiment are executed in Matlab/Simulink environment and laboratory sized overhead crane test-bed, respectively. Let consider the overhead crane with the nominal parameters are shown in Table 1.

Table 1. Nominal parameters of an overhead crane system

\begin{tabular}{ll}
\hline Items & Values \\
\hline Trolley mass $(M)$ & $25(\mathrm{~kg})$ \\
Payload mass $(m)$ & $8(\mathrm{~kg})$ \\
Cable length $(l)$ & $1.2(\mathrm{~m})$ \\
Gravity acceleration $(g)$ & $9.81\left(\mathrm{~m} / \mathrm{s}^{2}\right)$ \\
\hline
\end{tabular}


Based on the nominal parameters given in Table 1, the system model (4) can be rewritten as

$$
\left\{\begin{array}{l}
\dot{x}_{1}=x_{2} \\
\dot{x}_{2}=2.45 x_{3}+0.25 x_{3} x_{4}^{2}+0.05 u \\
\dot{x}_{3}=x_{4} \\
\dot{x}_{4}=-12.26 x_{3}-0.25 x_{3} x_{4}^{2}-0.05 u
\end{array}\right.
$$

The state-space model (11) with system uncertainties becomes

$$
\dot{x}=(A+\Delta A) x+B\left[u_{1}+\delta f(x, u)\right]
$$

where $A=\left[\begin{array}{cccc}0 & 1 & 0 & 0 \\ 0 & 0 & 2.45 & 0 \\ 0 & 0 & 0 & 1 \\ 0 & 0 & -12.26 & 0\end{array}\right], \quad B=\left[\begin{array}{c}0 \\ 0.05 \\ 0 \\ -0.25\end{array}\right]$

By solving (22) with $\zeta=0.5 k_{5}, Q=2 I$, and $R=5 \mathrm{e}-3 I$, the controller gain is obtained as:

$$
K=\left[\begin{array}{llll}
93.98 & 106.71 & -331.14 & 3.17
\end{array}\right]
$$

which leads to the following controller

$$
u_{1}=-K x-\delta_{e s} f(x, u)
$$

where $f(x, u)=u_{1}+x_{3} x_{4}^{2}, \quad \dot{\delta}_{e s}=\gamma f(x, u) x^{T} P B$ and $\gamma=0.05$. The overall controller:

$$
u=u_{1}+u_{2}
$$

where $u_{2}$ is shown in (5).

In order to verify the effectiveness of the adaptation component, the performances of the proposed controller are compared with the performances of the conventional state feedback controller via simulation and experimental results. The equations of the conventional state feedback controller are given by:

$$
\left\{\begin{array}{l}
u=u_{1}+u_{2} \\
u_{1}=-K_{f} x \\
u_{2}=-x_{3} x_{4}^{2}
\end{array}\right.
$$

where $K$ is calculated from nominal matrix $A$ and $B$ :

$$
K_{f}=\left[\begin{array}{llll}
1.4 & 1461.5 & -1461.3 & 290.6
\end{array}\right]
$$

In the paper, the simulations are carried out under three cases as follow:

Case 1: The system parameters are nominal, i.e., $M=25 \mathrm{~kg}, m=8 \mathrm{~kg}$, and $l=1.2 \mathrm{~m}$.

Case 2: The system parameters are of $150 \%$ variation, i.e., $M=37 \mathrm{~kg}, m=12 \mathrm{~kg}$, and $l=1.8 \mathrm{~m}$.

Case 3: The system parameters are nominal, i.e., $M=25 \mathrm{~kg}, m=8 \mathrm{~kg}, l=1.2 \mathrm{~m}$, and the input disturbance is $10 \sin (10 \mathrm{t})$.

In each case, the responses of the proposed algorithm is compared with the results of the state feedback controller. The simulation results for the above three cases are shown in Figure 2-4. In each figure, from top to bottom are the waveforms of the trolley position and payload swing angle, respectively. It can be seen from Figure 2 that, when the system parameters are nominal, the responses of the proposed scheme and the state feedback controller are not so much different. the settling time of the system with adaptive controller is about $3 \mathrm{sec}$, the tracking error is almost zero, and the maximum payload swing angle is $0.15 \mathrm{deg}$ (after $3 \mathrm{sec}$, the swing angle is cancelled). Meanwhile, the state feedback controller has the settling time about $5 \mathrm{sec}$ with no steady state error and $0.15 \mathrm{deg}$ of payload swing angle.

In the Figure 3, the system parameters are of $150 \%$ variation but the results of the proposed system are nearly unchanged, i.e. the transient time is less than $4 \mathrm{sec}$, the maximum swing angle is smaller than $0.15 \mathrm{deg}$ and it is kept almost zero at the steady state. For the state feedback controller, the response of the position is little oscilation with the longger settling time, about $6 \mathrm{sec}$, swing angle is still small $(0.2 \mathrm{deg})$ but it is underdamped oscillations. 
Figure 4 illustrates the responses of the proposed adaptive controller and state feedback controller in the presence of the input disturbance. It can be seen that, with the adaptive controller, the trolley reaches the destination after $3 \mathrm{sec}$ and the payload swing angle is removed after the transient time. However, in the case of the state feedback controller, the settling time is $5 \mathrm{sec}$ and the swing in the both position and payload angle is not cancelled although the trolley arrives its target.

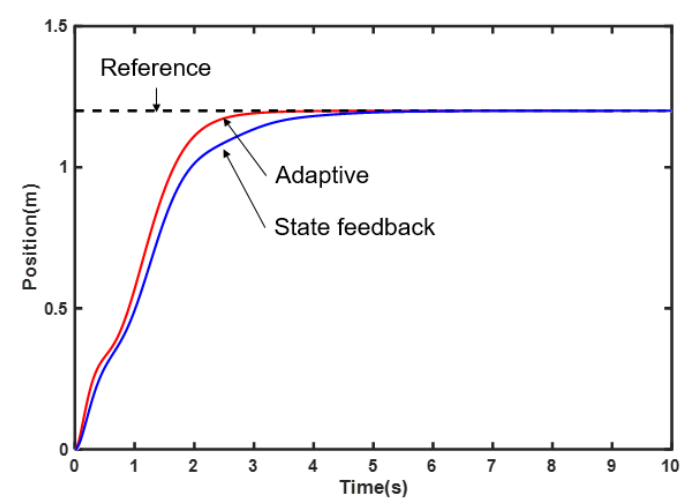

(a)

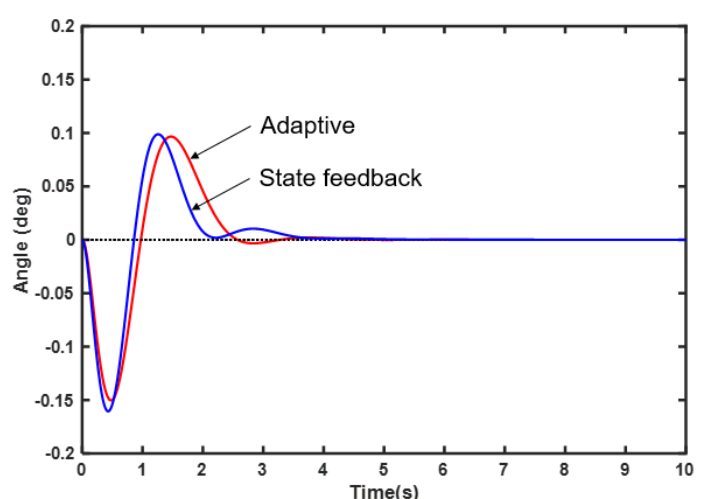

(b)

Figure 2. Simulation results of the proposed adaptive controller and state feedback controller with nominal system parameters, (a) trolley position, (b) payload swing angle

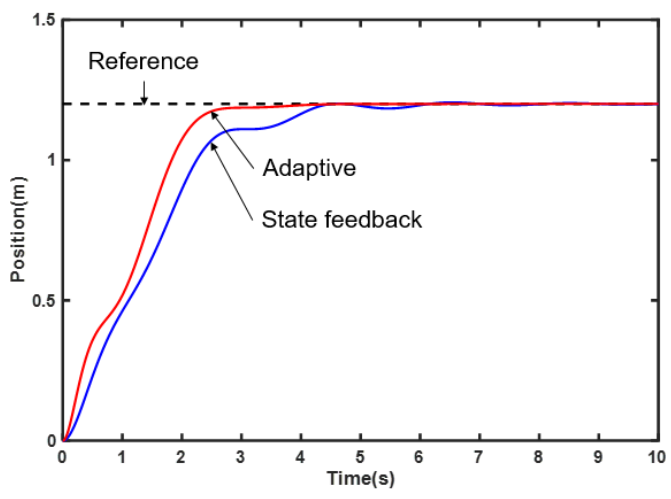

(a)

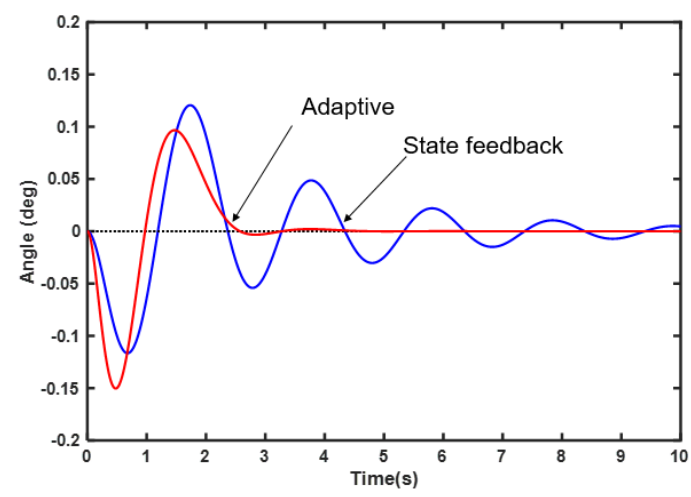

(b)

Figure 3. Simulation results of the proposed adaptive controller and state feedback controller with $150 \%$ variation of system parameters, (a) trolley position, (b) payload swing angle

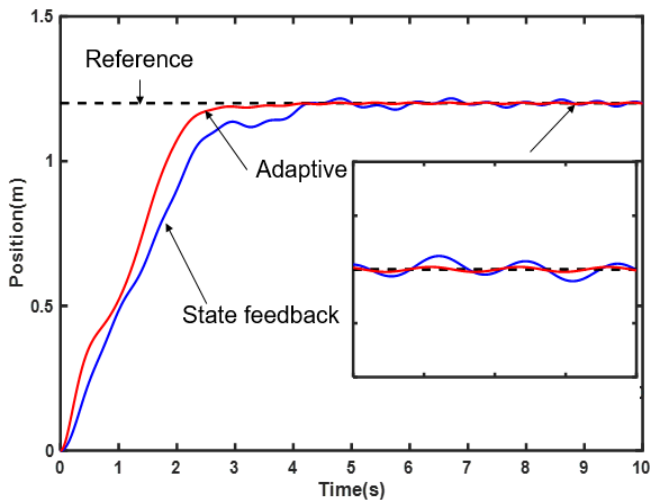

(a)

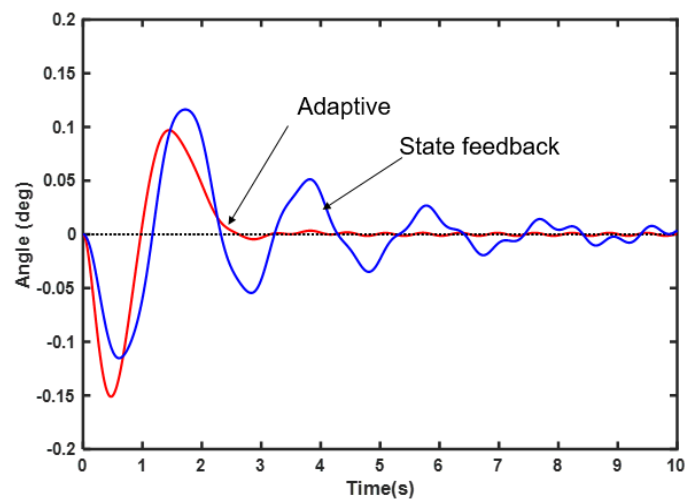

(b)

Figure 4. Simulation results of the proposed adaptive controller and state feedback controller with presence of the input disturbance, (a) trolley position, (b) payload swing angle 
The numerical analysis for above results is depicted in the Table 2. From the above simulation results, it is obvious that, the proposed adaptive controller and the corresponding state feedback controller keep the trolley stable at the destination. However, as the trolley mass, payload mass, and the cable length are changed or the input disturbance is sinusoidal, the suggested controller gives the performance with no redundant swing after the trolley comes to rest. Meanwhile, under the control of the state feedback scheme, the payload keeps swinging even though the trolley reaches the standstill condition.

Table 2. Position and angle responses of the proposed and state feedback controllers in three cases

\begin{tabular}{|c|c|c|c|c|c|c|}
\hline & \multicolumn{2}{|c|}{ Case 1} & \multicolumn{2}{|c|}{ Case 2} & \multicolumn{2}{|c|}{ Case 3} \\
\hline & Position & Angle & Position & Angle & Position & Angle \\
\hline Proposed & $\begin{array}{c}\mathrm{T}_{\mathrm{s}}=3 \mathrm{~s} \\
\text { No ripple }\end{array}$ & $\begin{array}{c}\mathrm{T}_{\mathrm{s}}=3 \mathrm{~s} \\
\text { No ripple } \\
\text { Max. }-0.15^{\circ}\end{array}$ & $\begin{array}{c}\mathrm{T}_{\mathrm{s}}=3 \mathrm{~s} \\
\text { No ripple }\end{array}$ & $\begin{array}{c}\mathrm{T}_{\mathrm{s}}=3 \mathrm{~s} \\
\text { No ripple } \\
\text { Max. }-0.15^{\circ}\end{array}$ & $\begin{array}{c}\mathrm{T}_{\mathrm{s}}=3 \mathrm{~s} \\
\text { No ripple }\end{array}$ & $\begin{array}{c}\mathrm{T}_{\mathrm{s}}=3 \mathrm{~s} \\
\text { No ripple } \\
\text { Max. }-0.15^{\circ}\end{array}$ \\
\hline $\begin{array}{c}\text { State } \\
\text { Feedback }\end{array}$ & $\begin{array}{c}\mathrm{T}_{\mathrm{s}}=5 \mathrm{~s} \\
\text { No ripple }\end{array}$ & $\begin{array}{c}4 \mathrm{~s} \\
\text { No ripple } \\
\text { Max. }-0.15^{\circ}\end{array}$ & $\begin{array}{c}\mathrm{T}_{\mathrm{s}}=5 \mathrm{~s} \\
\text { Little ripple }\end{array}$ & $\begin{array}{c}\mathrm{T}_{\mathrm{s}}>10 \mathrm{~s} \\
\text { Ripple } \\
\text { Max. }-0.13^{\circ}\end{array}$ & $\begin{array}{l}\mathrm{T}_{\mathrm{s}}=5 \mathrm{~s} \\
\text { Ripple }\end{array}$ & $\begin{array}{c}\mathrm{T}_{\mathrm{s}}>10 \mathrm{~s}, \\
\text { Ripple } \\
\text { Max. }-0.12^{\circ}\end{array}$ \\
\hline
\end{tabular}

\section{CONCLUSION}

A simple but efficient antisway adaptive controller has been presented for the overhead crane system. This simple controller not only removes the oscillation of the payload but it is also robust to the system uncertainties. Also, the linear matrix inequalities (LMI) with feasible performance constraints have been used to design the controller gains. The stability of the overall system was guaranteed by the Lyapunov theory. Finally, the simulation and was executed by Matlab/Simulink for both proposed adaptive controller and the state feedback controller. The simulation results indicate that the suggested controller gives the good performance, i.e., fast response, no steady state error, no payload swing angle even under the condition of system uncertainties.

\section{REFERENCES}

[1] K. C. C. Peng, et al., "Hand-motioncrane control using radio-frequency real-time location systems," IEEE/ASME Transactions on Mechatronics, vol. 17, no. 3, pp. 464-471, 2012.

[2] Y. G. Sung and W. E. Singhose, "Robustness analysis of input shaping commands for two-mode flexible systems," IET Control Theory and Applications, vol. 3, no. 6, pp. 722-730, 2009.

[3] N. Q. Hoang, et al., "Trajectory planning for overhead crane by trolley acceleration shaping," Journal of Mechanical Science and Technology, vol. 28, pp. 2879-2888, 2014.

[4] S. Garrido, et al., "Anti-swinging input shaping control of an automatic construction crane," IEEE Transaction on Automation Science and Engineering, vol. 5, no. 3, pp. 549-557, 2008.

[5] H. H. Lee, "Motion planning for three-dimensional overhead cranes with high-speed load hoisting," International Journal of Control, vol. 78, no. 12, pp. 875-886, 2005.

[6] N. Sun, et al., "A novel kinematic coupling-based trajectory planning method for overhead cranes," IEEE/ASME Transactions on Mechatronics, vol. 17, no. 1, pp. 166-173, 2012.

[7] B. d'Andréa-Novel, et al., "Finite-time stabilization of an overhead crane with a flexible cable," Mathematics of Control, Signals, and Systems, vol. 31, pp. 1-19, 2019.

[8] B. Lu, et al., "Adaptive output-feedback control for dual overhead crane system with enhanced anti-swing performance," IEEE Transactions on Control Systems Technology, pp. 1-14, 2019.

[9] M. R. Ghazali, et al., "An improved neuroendocrine-proportional-integral-derivative controller with sigmoidbased secretion rate for nonlinear multi-input-multi-output crane systems," Journal of Low Frequency Noise, Vibration and Active Control, 2019.

[10] X. Ma and H. Bao, "An Anti-Swing Closed-Loop Control Strategy for Overhead Cranes," Applied Sciences, vol. 8, no. 9 , pp. 1463, 2018.

[11] A. Cakan and U. Onen, "Position regulation and sway control of a nonlinear gantry crane system," International Journal of Scientific \& Technology Research, vol. 5, no. 11, pp. 121-124, 2016.

[12] X. Wu and X. He, "Partial feedback linearization control for 3-D underactuated overhead crane systems," ISA Transactions, vol. 65, pp. 361-370, 2016.

[13] T. A. Le, et al., "Partial feedback linearization and sliding mode techniques for 2D crane control," Transactions of the Institute of Measurement and Control, vol. 36, no. 1, pp. 78-87, 2014.

[14] B. Rong, et al., "Dynamics analysis and fuzzy anti-swing control design of overhead crane system based on Riccati discrete time transfer matrix method," Multibody System Dynamics, vol. 43, pp. 279-295, 2018.

[15] M. A. Shehu, et al., "Comparative Analysis of Neural-Network and Fuzzy Auto-Tuning Sliding Mode Controls for Overhead Cranes under Payload and Cable Variations," Journal of Control Science and Engineering, vol. 2019, pp. 1-13, 2019. 
[16] X. Shao, et al., "Takagi-Sugeno Fuzzy Modeling and PSO-Based Robust LQR Anti-Swing Control for Overhead Crane," Mathematical Problems in Engineering, vol. 2019, pp. 1-14, 2019.

[17] L. Ranjbari, et al., "Designing precision fuzzy controller for load swing of an overhead crane," Neural Computing and Applications, vol. 26, pp. 1555-1560, 2015.

[18] B. Lu, et al., "Sliding mode control for underactuated overhead cranes suffering from both matched and unmatched disturbances," Mechatronics, vol. 47, pp. 116-125, 2017.

[19] D. Chwa, "Sliding-Mode-Control-based robust finite-time antisway tracking control of 3-D overhead cranes," IEEE Transactions on Industrial Electronics, vol. 64, no. 8, pp. 6775-6884, 2017.

[20] Q. H. Ngo, et al., "Payload pendulation and position control systems for an offshore container crane with adaptivegain sliding mode control," Asian Journal of Control, pp. 1-10, 2019.

[21] T. Wang, et al., "A novel anti-swing positioning controller for two-dimensional bridge crane via dynamic sliding mode variable structure," Procedia Computer Science, vol. 131, pp. 626-632, 2018.

[22] C. Y. Chang, "Adaptive fuzzy controller of the overhead cranes with nonlinear disturbance," IEEE Transactions on Industrial Informatics, vol. 3, no. 2, pp. 164-172, 2007.

[23] M. S. Park, et al., "Antisway tracking control of overhead cranes with system uncertainty and actuator nonlinearity using an adaptive fuzzy sliding-mode control," IEEE Transactions on Industrial Electronics, vol. 55, no. 11, pp. 3972-3984, 2008.

[24] M. S. Park, et al., "Adaptive sliding-mode antisway control of uncertain overhead cranes with high-speed hoisting motion," IEEE Transactions on Fuzzy Systems, vol. 22, no. 5, pp. 1262-1271, 2014.

[25] H. Chen, et al., "Optimal trajectory planning and tracking control method for overhead cranes," IET Control Theory and Applications, vol. 10, no. 6, pp. 692-699, 2016. 